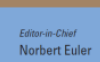

Norbert Euler

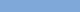

Journal of Nonlinear Mathematical Physics

ISSN (Online): 1402-9251 ISSN (Print): 1776-0852

Journal Home Page: https://www.atlantis-press.com/journals/jnmp

\title{
Preface to Special Issue on the Geometry of the Painlevé equations
}

Nalini Joshi, Masatoshi Noumi, Hidetaka Sakai, Claude M. Viallet

To cite this article: Nalini Joshi, Masatoshi Noumi, Hidetaka Sakai, Claude M. Viallet (2013) Preface to Special Issue on the Geometry of the Painlevé equations, Journal of Nonlinear Mathematical Physics 20: Supplement 1, 1-2, DOI:

https://doi.org/10.1080/14029251.2013.862429

To link to this article: https://doi.org/10.1080/14029251.2013.862429

Published online: 04 January 2021 


\title{
Preface to Special Issue on the Geometry of the Painlevé equations
}

\author{
Nalini Joshi \\ School of Mathematics and Statistics F07 \\ The University of Sydney, NSW 2006, Australia \\ nalini.joshi@sydney.edu.au \\ Masatoshi Noumi \\ Department of Mathematics, Kobe University \\ Rokko, Kobe 657-8501, Japan \\ noumi@math.kobe-u.ac.jp \\ Hidetaka Sakai \\ Graduate School of Mathematical Sciences, The University of Tokyo 381 \\ Komaba Meguro-ku, Tokyo 153-8914, Japan \\ sakai@ms.u-tokyo.ac.jp \\ Claude M. Viallet \\ LPTHE, Université Pierre et Marie Curie, Centre National de la Recherche Scientifique \\ UMR 7589 Boîte 126, 4 Place Jussieu, 75252 Paris Cedex 05, France \\ viallet@lpthe.jussieu.fr
}

This special issue focuses on the geometry of the space of initial values underlying the continuous Painlevé equations, which was initiated by Okamoto in 1979 [3]. The purpose of this issue is to review the area and report on the latest developments. As geometry is a broad word, we have also included possible extensions to the theory of monodromy groups and the linear equations associated with the Painlevé equations.

The Painlevé equations, which are integrable, classical ordinary differential equations, are now recognized as non-linear special functions of great importance in modern science. Their prominence in the applications of Random Matrix Theory has strengthened this impact.

The geometric theory initiated by Okamoto was extended and used to classify all continuous and discrete Painlevé equations by Sakai in 2001 [4]. Moreover, the underlying affine Weyl groups associated with the geometry of initial value spaces have been used to construct many other important properties, such as special solutions, by Noumi [2] and collaborators. Bellon and Viallet's [5] definition of algebraic entropy and its effectiveness as a test of integrability is based on geometric properties of such integrable systems. Duistermaat and Joshi [1] have illustrated the power of the geometric theory by showing how to deduce analytic information for solutions of the Painlevé equations from the geometric construction. However, the geometric theory remains little understood by most western mathematicians who work in integrable systems. The aim of this issue is to build a bridge between the geometric approach, primarily developed by Japanese mathematicians, and other approaches.

We hope this special issue will encourage a wide range of mathematicians to recognize the remarkable role played by the geometric approach to the Painlevé equations and integrable systems. 


\section{References}

[1] J.J. Duistermaat, N. Joshi, Okamoto's space for the first Painlevé equation in Boutroux coordinates. Archive for Rational Mechanics and Analysis, 202 (3) (2011) 707-785.

[2] M. Noumi, Painlevé Equations Through Symmetry. Translations of Mathematical Monographs, 223 AMS (2004).

[3] K. Okamoto: Sur les feuilletages associés aux équation du second ordre à points critiques fixes de $\mathrm{P}$. Painlevé. Espaces de conditions initiales. Japanese J. Math. 5 (1979) 1-79.

[4] H. Sakai, Rational surfaces associated with affine root systems and geometry of the Painlevé equations. Comm. Math. Phys. 220 (1) (2001) 165-229.

[5] M. P. Bellon and C.M. Viallet, Algebraic entropy, Comm. Math. Phys. 204 (2) (1999) 425-437. 